\title{
Low ionization lines in high luminosity quasars: The calcium triplet
}

\author{
Mary Loli Martínez-Aldama*, Deborah Dultzin \\ Instituto de Astronomía, Universidad Nacional Autónoma de México, Aptdo. Postal \\ 70-264, México, D. F. 04510, Mexico. \\ Paola Marziani \\ INAF, Osservatorio Astronomico di Padova, Vicolo dell Osservatorio 5, 35122 Padova, \\ Italy \\ Jack W. Sulentic \\ Instituto de Astronomía de Andalucía (CSIC), C/ Camino Bajo de Huétor 50, 18008 \\ Granada, Spain. \\ Yang Chen, Alessandro Bressan \\ Scuola Internazionale Superiore di Studi Avanzati (SISSA), via Bonomea 265, I-34136 \\ Trieste, Italy \\ Giovanna M. Stirpe \\ INAF, Osservatorio Astronomico di Bologna, via Ranzani 1, 40127 Bologna, Italy.
}

\begin{abstract}
In order to investigate where and how low ionization lines are emitted in quasars we are studying a new collection of spectra of the CaII triplet at $\lambda 8498, \lambda 8542, \lambda 8662$ observed with the Very Large Telescope (VLT) using the Infrared Spectrometer And Array Camera (ISAAC). Our sample involves luminous quasars at intermediate redshift for which CaII observations are almost nonexistent. We fit the CaII triplet and the OI $\lambda 8446$ line using the $\mathrm{H} \beta$ profile as a model. We derive constraints on the line emitting region from the relative strength of the CaII triplet, OI $\lambda 8446$ and $\mathrm{H} \beta$.
\end{abstract}

\footnotetext{
${ }^{*}$ Corresponding author

Email address: maldama@astro.unam.mx (Mary Loli Martínez-Aldama)
} 
Keywords: Line formation; Quasars: general; Quasars: emission lines

\section{Introduction}

Explaining the origin of Fe emission in quasars is a long-standing problem in active galactic nuclei (AGN) research. The extreme complexity of the FeII ion makes theoretical model calculations very difficult and line blending makes estimation of FeII width and strength parameters uncertain. The $\mathrm{Ca}^{+}$ ion is, by contrast, far simpler. The ionization potential of neutral Calcium is $6.1 \mathrm{eV}$ so we expect CaII ions to exist wherever hydrogen is not fully ionized. Several lines of evidence suggest that CaII IR triplet at $\lambda 8498, \lambda 8542, \lambda 8662$ (hereafter CaII for brevity) and optical FeII are produced in the same region. Data from Persson (1988) and photoionization calculations from Joly (1989) found that CaII is emitted by gas with low temperature $(8000 \mathrm{~K})$, high density $\left(>10^{11} \mathrm{~cm}^{-3}\right)$ and a high column density $\left(>10^{23} \mathrm{~cm}^{-2}\right)$ similar to the optical FeII. Ferland and Persson (1989) improved the photoionization models including physical processes like $\mathrm{H}^{0}$ free-free, $\mathrm{H}^{-}$bound-free and Compton recoil ionization, that showed the need for very large column densities $\left(>10^{24.5} \mathrm{~cm}^{-2}\right)$ to reproduce the CaII spectrum. Such large column density gas could be provided by an accretion disk. A similar behavior for $\mathrm{CaII} / \mathrm{H} \beta$ and $\mathrm{FeII} / \mathrm{H} \beta$ was found by Dultzin-Hacyan et al. (1999). These authors also suggested that the line emitting region could be associated with the outer part of an accretion disk. Recently Matsuoka et al. (2007) performed photoionization models and found that low ionization parameters ( $\mathrm{U}$ $\sim 10^{-2.5}$ ) are needed to reproduce the flux ratios. Therefore, there is evidence pointing toward low ionization lines like FeII and CaII arising in a region probably associated with an accretion disk, with physical conditions different from the regions emitting most of the high ionization lines.

The present work extends the study of CaII $\lambda 8498, \lambda 8542, \lambda 8662$ to 14 high luminosity and intermediate redshift quasars that are analyzed within the $4 \mathrm{D}$ Eigenvector 1 parameter space context (4DE1; Sulentic, Marziani \& DultzinHacyan, 2000; Sulentic et al., 2000; Marziani et al., 2001, 2003a,b; Sulentic et al., 2007), which serves as a spectroscopic unifier/discriminator of the emission lines properties for type 1 AGN. 4DE1 includes (1) FWHM $\left(\mathrm{H} \beta_{B C}\right)$, (2) equivalent width (EW) of the optical Fe II $\lambda 4570$ blend and $\mathrm{H} \beta$ defined as the ratio $\mathrm{R}_{F e I I}=\mathrm{W}(\mathrm{Fe}$ II $\lambda 4570) / \mathrm{W}(\mathrm{H} \beta)$, (3) the soft X-ray photon index 
$\left(\Gamma_{\text {soft }}\right)$ and (4) the centroid line shift of high-ionization C IV $\lambda 1549$ with the strongest correlations involving parameters 2, 3 and 4 (Sulentic et al., 2007).

In the 4DE1 parameter framework the broad line AGNs can be divided in two populations, A and B (Sulentic et al., 2002). Considering the broad component of the $\mathrm{H} \beta$ line, population $\mathrm{A}$ and $\mathrm{B}$ can be separated at $\mathrm{FWHM}\left(\mathrm{H} \beta_{B C}\right)$ $=4000 \mathrm{~km} \mathrm{~s}^{-1}$; spectra of sources above and below this limit look substantially different. Population A shows: (1) a scarcity of RL sources, (2) strong/moderate Fe II emission, (3) a soft X-ray excess, (4) high-ionization broad lines with blueshift/asymmetry and (5) low-ionization broad line profiles (LIL) best described by Lorentz fits. Meanwhile, Population B: (1) includes the large majority of the RL sources, (2) shows weak/moderate Fe II emission, includes sources (3) with less prominent or no soft X-ray excess (Sulentic et al., 2007) and (4) with HIL blueshift/asymmetry or no blue shifts at all. Last, (5) Pop. B shows LIL Balmer lines best fit with double Gaussian models. The physical drivers that change along the 4DE1 sequence have been identified: number density appears to increase from Pop. B to A, and black hole mass increases with large scatter from A to B. The principal driver of source occupation in 4DE1 space involves Eddington ratio which increases from B to A (Marziani et al., 2001).

This paper presents new observations and data reduction of spectra of the CaII IR triplet (§2), as a representative low ionization lines, which are used to try to know the physical conditions from the emitting region. We discuss in some detail the line and continuum components identified for a proper data analysis (§3). Preliminary results obtained for the comparison between observational data and photoionization predictions are briefly reported in $\S 4$. Conclusions are present in $\S 5$.

\section{Observations and data reduction}

\subsection{Sample selection and observations}

Up to recent time CaII samples were selected in sources with strong FeII emission and low redshift (Persson, 1988; Matsuoka et al., 2007). CaII had been seen in absorption, coming from the host galaxy of the AGN, so that a strong FeII emission guarantees the observation of CaII in emission from the broad line region (BLR). Our sample was selected without considering FeII intensity. We selected sources where CaII and OI lines were not affected by 
atmospheric absorption present in the infrared region. Also, we only chose targets where the optical spectrum around $\mathrm{H} \beta$ has already been observed.

Following the 4DE1 context, population A sources show strong FeII emission and therefore we expect CaII emission to be strong. The majority of previous observations involved population A sources where both ions show strong emission. Our sample contains 4 Pop. A and 10 B sources making lower intensity FeII sources well represented in our sample. Our sample therefore explores a domain in FeII emission where the study of CaII could give new hints about the origin of low ionization lines.

The sample contains 14 high luminosity Hamburg-ESO quasars with a spectral resolution, $\mathrm{R}_{S}, \sim 300, \mathrm{M}_{B}<-26$ and $0.847<z<1.638$ observed at the Very Large Telescope (VLT) equipped with the Infrared Spectrometer And Array Camera (ISAAC) during 2010, in service mode. Table 1 lists target name, redshift, absolute magnitude, Kellermann's coefficient for radioloudness and spectral type in the Eigenvector 1 sequence. The last column reports the standard star name used for sensitivity function calibration.

VLT is made up by four telescopes of $8.2 \mathrm{~m}$ diameter located on top of Cerro Paranal. Each telescope operates with a large collection of high quality instruments. ISAAC is one of them, and has been for many years one of the few instruments available worldwide for moderate resolution IR spectroscopy of faint sources like high- $z$ quasars. It is a camera able to obtain images and spectra with a high resolution at $1-5 \mu \mathrm{m}$ (Moorwood et al., 1998) and it is equipped with gratings for high $\left(\mathrm{R}_{S} \sim 3000\right)$ and low resolution $\left(\mathrm{R}_{S} \sim 500\right)$ for a 1" slit width. Our spectra were collected at low resolution but with a slit width of 0.6 " that ensured a spectral resolution $\mathrm{R}_{S} \approx 1000$.

\subsection{CaII - data reduction}

Data reductions were performed using IRAF software. Sequences of frames were obtained alternating the source placement at different locations (e.g. A, B) along the slit. All frames with the same source location were averaged. The average observation at one location was subtracted from the one at the different position to obtain two background subtracted frames (e. g. $\bar{A}-\bar{B}$, and $\bar{B}-\bar{A}$ ). The resulting differences were divided by the appropriate flat field frames. Spectra were extracted using the IRAF program apsum and 
were calibrated on wavelength with a xenon/argon arc spectrum that was extracted from the calibration frame. The wavelength scale was set using 3rd order Chebyshev polynomial fits to the positions of the most intense lines in the $\mathrm{H}$ and $\mathrm{K}$ band, $1.5-2 \mu \mathrm{m}$ and $2-3 \mu \mathrm{m}$ respectively. Once matched with the corresponding arc calibrations, spectra of each source were rebinned to a linear wavelength scale whose zero point was readjusted using suitable skylines. Frames were then averaged with weights proportional to their total integration time.

The spectra of the telluric standard stars were extracted and wavelengthcalibrated in the same way. We eliminated the atmospheric features by dividing the quasar and the standard star spectra by a synthetic atmospheric transmission spectrum. The majority of the standard stars are spectral type B. Unfortunately, we could not find theoretical libraries of spectra with a consistent model for reproducing the spectrum in the $\mathrm{H}$ and $\mathrm{K}$ band. Therefore we decided to use a black-body model corresponding to the temperature of the star determined on the basis of its tabulated spectral type, since this approach yielded a good approximation to stars with observed IR spectral energy distribution. The sensitivity function was then obtained dividing the standard star spectrum by the black-body model.

Finally, the correct flux calibration was achieved by scaling the standard star spectra according to its magnitude. Since the seeing almost always exceeded the width of the slit, a significant light loss occurred and an additional correction had to be applied. Although extinction is not large in this region, the spectra were corrected using the data given by Schlegel, Finkbeiner \& Davis (1998). Redshift correction was carried out assuming redshift values reported by Sulentic et al. (2004) and Marziani et al. (2009) based on $\mathrm{H} \beta_{N C}$, $\mathrm{H} \gamma_{N C}$ and [OIII] $\lambda \lambda 5007,4959$ with an uncertainty usually $<150 \mathrm{~km} \mathrm{~s}^{-1}$. The right-hand panels of Figure 1 show examples of two calibrated CaII spectra.

\section{3. $H \beta$ observations}

CaII observations are complemented with optical spectra that cover all or part of the $\mathrm{H} \beta$, FeII $\lambda 4570$ and/or FeII $\lambda 5130$ blend. Optical measurements were obtained with VLT ISAAC in service mode between 2001 and 2005 (Sulentic et al., 2004; Marziani et al., 2009). Since H $\beta$ and CaII spectra were observed at widely different epochs $(\approx 10 \mathrm{yr}$ apart $)$, both spectra 
were normalized to 2MASS magnitudes, setting a normalized flux scale of the same epoch to avoid variability effects (the response times of the BLR to continuum changes is several years). HE0048-2804 and HE2340-4443 were observed in the $\mathrm{Z}$ band to cover $\mathrm{H} \beta$. 2MASS data are not available in $\mathrm{Z}$, so that no normalization was applied to their $\mathrm{H} \beta$ spectra. The flux scale is therefore the one obtained from the standard star. Two examples of $\mathrm{H} \beta$ are shown in the left panels of Figure 1.

\section{Data analysis}

We built models of the spectral regions using specfit, an IRAF task that preforms simultaneous fits of various line and continuum components (Kriss, 1994). Each component has a specific number of associated parameters. Providing a lower and an upper limit, and an initial guess value for each parameter, specfit can compute a minimum $\chi^{2}$ fit. At first we performed the $\mathrm{H} \beta$ fits, allowing all parameters to vary freely. Although Sulentic et al. (2004) and Marziani et al. (2009) provide previous ones for $\mathrm{H} \beta$, we decided to redo all measurements for $\mathrm{H} \beta$ as aid for processing high order Paschen lines, where the same FWHM and shift were fitted. For CaII and OI we only took the $\mathrm{H} \beta$ FWHM as an initial guess, leaving specfit free to compute the best model within reasonable upper and lower limits. Two examples of the fits are shown in Figure 2 (left: $\mathrm{H} \beta$, right: $\mathrm{CaII}$ ). In the following we describe the component used for the fits.

1. Continuum. We assume that the continuum underlying optical and near-infrared regions is a power law with variable slope. For $\mathrm{H} \beta$ it was fixed taking as reference 4750 and $5100 \AA$. Due to the small bandwidth of our IR spectra, the identification of the continuum was difficult. Depending on the wavelength range in each spectrum, we fit a local continuum taking one or two reference points at 8100, 8800 and 9400 A, obtaining a different slope for each case.

2. FeII template. For the FeII optical contribution in $\mathrm{H} \beta$ we used the template previously employed by Marziani et al. (2009). In the past, the main efforts in modelling the FeII contribution were focussed on the ultraviolet and optical regions. Only recently Garcia-Rissmann et al. (2012) produced the first semi-empirical near-infrared template based 
on the I Zw 1 spectrum. We used the template kindly made available to us by Garcia-Rissmann et al. (2012) to model the FeII emission. The main FeII contributions are at $9200 \AA$ close to Pa9 $\lambda 9229$. According to Garcia-Rissmann, in I Zw 1 the contribution is $\sim 50 \%$ of Pa9. This relative intensity is however not always observed since there are objects in which FeII is visually stronger as, for example, HE0248-3628. We also found a slight FeII deficit in the blue side of Pa9. In those cases we preferred to use the theoretical values reported in the paper by Garcia-Rissmann et al. (2012), and found that the fits improved. In several spectra we observe a little bump at 8200-8300 $\AA$ that cannot be reproduced using either the theoretical or the semi-empirical template.

3. Broad component. Following 4DE1 we assume that low ionization lines in Pop. A and B sources (FWHM H $\beta_{B C}<$ and $>4000 \mathrm{~km} \mathrm{~s}^{-1}$ respectively) show Lorentzian and double Gaussian profiles in the broad component. Therefore all the broad components were fitted using a Lorentzian in Pop. A sources and a Gaussian in Pop. B sources. The $\mathrm{H} \beta$ line was taken as a reference to carry out the fits of the CaII blend, since its profile is well defined. The $\mathrm{H} \beta$ FWHM and shifts were also applied to high order Paschen lines present in the near-infrared spectra. Even if $\mathrm{H} \beta$ and $\mathrm{OI}$ are emitted in a region with similar physical conditions (see below), the FWHM is not the same: $\mathrm{FWHM}\left(\mathrm{OI}_{B C}\right) \leq$ FWHM $\left(\mathrm{H} \beta_{B C}\right)$, although OI FWHM was allowed to vary over a wide range. CaII triplet was modelled using three BCs of equal intensity and with the same FWHM, since the CaII triplet is optically thick (Ferland and Persson, 1989). As CaII lines are completely blended it is difficult to measure a shift, and so the CaII lines were held fixed at rest-frame wavelength.

4. Very broad component. A red asymmetry in population B sources with $\mathrm{H} \beta \quad \mathrm{FWHM} \gtrsim 4000 \mathrm{~km} \mathrm{~s}^{-1}$ has been associated with the existence of a distinct emitting region, the so-called Very Broad Line Region (VBLR; Marziani et al., 2009). A VBC was fit to $\mathrm{H} \beta$, OI $\lambda 8446$ and the most intense high order Paschen lines: Pa9, Pa8 and Pa7. The shift and FWHM were assumed equal in $\mathrm{H} \beta$ and Paschen lines. Simulation results indicate that $\mathrm{OI}$ and $\mathrm{H} \beta$ are emitted in similar regions, whose physical conditions are less restrictive than those needed for significant CaII emission (see §4.1). Therefore a VBC was not fitted to CaII emission.

5. Narrow component. The narrow lines or components present in the 
optical spectra are [OIII] $\lambda \lambda 5007,4959$ and $\mathrm{H} \beta$. Due to the low $\mathrm{S} / \mathrm{N}$ in near-infrared spectra it is usually not possible to detect narrow line emission (that is anyway weak in most cases): only in HE1349+0007 we were able to fit the NC of OI. We could also detect [SIII] $\lambda 9531$.

6. High order Paschen lines. The most intense Paschen lines in our spectra are $\mathrm{Pa} 9, \mathrm{~Pa} 8$ and $\mathrm{Pa} 7$. However, if we only considered these lines along with FeII, we would get a deficit of emission at 8700-9100 $\AA$, so we have decided to include high order Paschen lines. Because Pa9 is present in all our spectra, we decided to take it as a reference. Higher order Paschen lines were scaled using the results of CLOUDY simulations (version 08.00) (Ferland et al., 1998). We noted the presence of the high order Paschen lines form a pseudo-continuum that cannot be neglected. This pseudo-continuum covers part of the CaII triplet region specially at $\lambda 8662$. Its inclusion has an effect on the intensity and FWHM of the CaII triplet. Given that our objective is to try to model the emission in this region as accurately as possible, we decided to consider high order Paschen lines from Pa9 to Pa24. Persson (1988) found that Pa14 $\lambda 8598$ contributes $\sim 12 \%$ of the flux of $\lambda 8662$ in Mrk 42 , so it was ignored. However in HE0035-2853, we found that the contribution of Pa14 is 70\%, while in HE0048-2804 CaII emission is insignificant and Paschen lines dominate the fit.

7. Paschen continuum. Two of our targets, HE0058-3231 and HE22022557 cover the head of the Paschen continuum $(\mathrm{PaC})$ at $8204 \AA$. It is interesting to note that previous quasar studies have neglected the $\mathrm{PaC}$ due to the expectation that its contribution would be smaller than the Balmer continuum. We could detected a hint of PaC in HE0058-3231 and HE2202-2557. In the other sources we could only cover the region beyond the Paschen limit. A CLOUDY simulation with $\log (U)=-2.5$ and $\log \left(n_{\mathrm{H}}\right)=12$ provided us with predictions on the relative intensity of Pa9 and of the integrated PaC (c.f. Osterbrock \& Ferland, 2005). We then estimated the continuum specific intensity at the Paschen edge, and assumed an exponential decrease toward shorter wavelengths appropriate for an optically thin continuum. Our estimates appear to be in excess with respect to the observations since the best fits require a $\mathrm{PaC}$ smaller than that predicted. There are two main possible explanations: 1) CLOUDY computations overpredict the recombination continuum, or 2) the quasar continuum level we set is not correct; the actual continuum is lower. Unfortunately, we cannot test these options 
on our data since the spectra cover only a small wavelength range. To properly define $\mathrm{PaC}$ we need to cover a spectral range spanning from the optical to the NIR. We will try to address the issue of the $\mathrm{PaC}$ intensity in the future.

8. Stellar absorptions. To subtract the stellar component we used new stellar population synthesis models (Chen et al. in preparation), based on the code of Bressan, Granato \& Silva (1998) with updated stellar evolutionary tracks (Bressan et al., 2012) and stellar atmospheres suitable for the analysis of stellar absorption lines in the optical and near infrared spectral regions (Sánchez-Blázquez et al., 2006a,b,c; Rayner et al., 2009). We found that the underlying stellar absorption of the host galaxy is significant only in HE2202-2557, with a luminosity contribution of $\sim 20 \%$, while the rest of the sample was affected only by $<10 \%$. The stellar population synthesis model was selected taking into account redshift (that sets the maximum age of the host galaxy) and typical black hole mass: we assumed a spheroid mass of $1.13 \times 10^{12} \mathrm{M}_{\odot}$, an age of $2.4 \mathrm{Gyr}$, and metallicity of $2 \mathrm{Z}_{\odot}$. These properties are consistent with massive ellipticals expected to host very luminous quasars at intermediate redshift.

\section{Preliminary results}

\subsection{Observations vs. photoionization models}

If we consider the broad component only, the flux ratios in our sample are approximately $\log (\mathrm{CaII} / \mathrm{OI}) \approx 0.01, \log (\mathrm{CaII} / \mathrm{H} \beta) \approx-0.7, \log (\mathrm{CaII} / \mathrm{Pa} 9)$ $\approx 0.35, \log (\mathrm{OI} / \mathrm{H} \beta) \approx-0.8$ and $\log (\mathrm{OI} / \mathrm{Pa} 9) \approx 0.1$. We carried out model calculations in the photoionized BLR using the code CLOUDY version 08.00 (Ferland et al., 1998). The gas was modeled with a column density of $10^{23}$ $\mathrm{cm}^{-2}$ and $\left(\mathrm{U}, \mathrm{n}_{H}\right)$ sets of $7<\log n_{H}<13$ and $-4.5<\log U<0$. Chemical composition was assumed solar. The measured ratios, compared to those predicted by the simulations, consistently indicate $\mathrm{n}_{H}>10^{11.5} \mathrm{~cm}^{-3}$ for CaII in agreement with previous works (Joly, 1989; Ferland and Persson, 1989; Matsuoka et al., 2007, 2008) and with the physical conditions found for FeII. The ionization parameter is constrained to be $\log U<-1.5$.

\subsection{Where are OI and CaII emitted?}

OI emission is favored at somewhat lower density $\left(\mathrm{n}_{H}<10^{11} \mathrm{~cm}^{-3}\right)$ and higher ionizing photon flux than CaII; in other words, the behaviour of OI 
is more similar to that of $\mathrm{H} \beta$. Since $\mathrm{H} \beta$ and OI are expected to be emitted in similar regions on the basis of photoionization predictions, we include a $\mathrm{VBC}$ for the OI line of population B sources. This means that emission of $\mathrm{H} \beta$ and OI are assumed to occur in both a Broad Line Region and a Very Broad Line Region associated with the BC and VBC respectively. A good fit to the profile blend $\mathrm{OI}+\mathrm{CaII}$ is possible in this case. The resulting OI profile is consistent with $\mathrm{H} \beta \mathrm{BC}+\mathrm{VBC}$ and broader than CaII for which we include a $\mathrm{BC}$ only. We stress again that CaII is expected to be emitted mainly in the $\mathrm{BLR}$ associated with the $\mathrm{BC}$ only, since the ionization parameter and density in the VBLR are believed to be unsuitable for significant CaII emission.

Combining our data and Persson's we can see that the original relation between FWHM of CaII, OI $\lambda 8446$ and $\mathrm{H} \beta$ found by Persson (1988) himself is basically confirmed: $\operatorname{FWHM}\left(\mathrm{H} \beta_{B C}\right) \sim \operatorname{FWHM}(\mathrm{CaII}) \sim \operatorname{FWHM}\left(\mathrm{OI}_{B C}\right)$ (Fig. 3). There are however a few Pop. A sources that have a significant broader CaII. Our sources are too low $\mathrm{S} / \mathrm{N}$ to claim that this difference is real. The data of Persson were analyzed without several emission features (FeII, OI narrow component, high order Paschen lines, ...) that we now know are blended with OI and CaII. To ascertain that FWHM(OI) $<$ FWHM(CaII) in Pop. A, a new analysis of the old Persson's data should be carried out.

On the other hand, taking the full profile $(\mathrm{BC}+\mathrm{VBC})$ in Pop. B sources for $\mathrm{H} \beta$ and OI, we obtained the following average values: $\mathrm{FWHM}\left(\mathrm{H} \beta_{B C+V B C}\right)$ $=6100 \mathrm{~km} \mathrm{~s}^{-1}, \operatorname{FWHM}\left(\mathrm{OI}_{B C+V B C}\right)=5500 \mathrm{~km} \mathrm{~s}^{-1}$ and $\operatorname{FWHM}(\mathrm{CaII})=$ $4600 \mathrm{~km} \mathrm{~s}^{-1}: \operatorname{FWHM}\left(\mathrm{H} \beta_{B C+V B C}\right)>\operatorname{FWHM}\left(\mathrm{OI}_{B C+V B C}\right)>\operatorname{FWHM}(\mathrm{CaII})$ (Figure 3 ). This second sequence reflects the likely possibility of an ionization stratification from the innermost zones where ionization is too high for OI and CaII emission, to the outermost ones where CaII emission if favored.

The equivalent width of CaII is distributed over a wide range, whereas the equivalent width of $\mathrm{OI}$ is not. This result also indicates that OI is unlikely to be emitted exclusively in the very same region of the CaII triplet. OI is pumped by Bowen fluorescence, but CaII is not and Ly $\beta$ photons can ionize $\mathrm{Ca}^{+}$to $\mathrm{Ca}^{++}$from $\mathrm{Ca}^{+}$ground level. Pumping is especially effective at the boundary between the fully and partially ionized zone (PIZ), where OI can be neutral. Therefore significant emission is expected whenever the Ly $\beta$ opacity is high and the gas is optically thick to the ionizing continuum, so that a PIZ can exist. Large column density is necessary. 
The previous results suggest that OI emission is not restricted to the CaII emitting region. We cautiously considered VBC emission of OI on the basis of appearance of the OI+CaII blends and of the photoionization simulations prediction, assuming that no OI VBC implies stronger CaII emission. Our conclusions will be little affected in this case because, at low ionization parameter, the CaII / Pa9 ratio shows a steep increase with density at $\log n_{H}>11-11.5$. Only if the CaII triplet were not detected at all, our conclusions would be affected. We plan, however, to study the effect of different emissivity laws on line profiles in a forthcoming study (Martínez-Aldama et al., in preparation).

Both CaII and OI are emitted in the BLR associated with the BC along with other higher ionization lines. We can figure out a configuration that is plane parallel with a distribution of clouds/filaments above and below the accretion disk. Some of the clouds might be at the same distance from the ionization source as the dense CaII emitting regions within the accretion disk. Therefore, clouds and disk may share the same dynamics but they do not necessarily share the same physical conditions. This provides an explanation for the similarity of the BC profiles; a slightly larger emissivity-weighted distance for OI could explain some FWHM differences, for example FWHM(CaII) > $\mathrm{FWHM}\left(\mathrm{OI}_{B C}\right)$.

\section{Conclusions}

We analyzed a new sample of CaII IR triplet and OI $\lambda 8446$ in luminous quasars at intermediate redshift in order to investigate the physical conditions required for low ionization line emission. This work extends previous samples including several quasars with broader lines (Pop. B) and modest or weak FeII emission. For the first time we include a FeII NIR template, high order Paschen lines and Paschen continuum in the analysis of the spectral region around CaII triplet. According to photoionization predictions, CaII is emitted in a region with $\mathrm{n}_{H}>10^{11.5} \mathrm{~cm}^{-3}$ and $\log U<-1.5$. These conditions are similar to those capable of accounting for the FeII emission in a photoionization scenario. Meanwhile OI emission is favored $\mathrm{n}_{H}<10^{11} \mathrm{~cm}^{-3}$ and higher ionizing photon flux. The physical conditions for OI are more similar to $\mathrm{H} \beta$, so that we included $\mathrm{BC}+\mathrm{VBC}$ emission in Pop. B., sources 
for OI $\lambda 8446$. This yielded a better fit of the whole OI+CaII blend.

We thank both reviewers for their useful comments to improve this manuscript. D.D. acknowledges support from Grant IN107313, PAPIIT UNAM.

\section{References}

Bressan, A., Granato, G. L., \& Silva, L., Modelling intermediate age and old stellar populations in the Infrared, 1998, A\&A, 332, pp. 135-148

Bressan, A., Marigo, P., Girardi, L., et al., PARSEC: stellar tracks and isochrones with the PAdova and TRieste Stellar Evolution Code, 2012, MNRAS, 427, pp. 127-145

Dultzin-Hacyan, D., Taniguchi, Y., Uranga, L., Where is the Ca II Triplet Emitting Region in AGN?, 1999, ASPC, 175, pp. 303-308

Ferland, G. J., Persson, S. E., et al., Implications of CA II emission for physical conditions in the broad-line region of active galactic nuclei, 1989, ApJ, 347, pp. 656-673

Ferland, G. J., Korista, K. T., Verner, D. A., et al., CLOUDY 90: Numerical Simulation of Plasmas and Their Spectra, 1998, PASP, 110, pp. 761-778

Garcia-Rissmann, A. Rodriguez-Ardila, A., Sigut, T. A. A., et al., A Nearinfrared Template Derived from I Zw 1 for the Fe II Emission in Active Galaxies, 2012, ApJ, 751, pp. 7-19

Joly, M., Formation of CA II lines in active galactic nuclei, 1989, A\&A, 208, pp. $47-51$

Kriss, G., Fitting Models to UV and Optical Spectral Data, 1994, Astronomical Data Analysis Software and Systems III, ASP Conf. Ser., 61, pp. 437-446

Marziani P., Sulentic J. W., Zwitter T., et al., Searching for the Physical Drivers of the Eigenvector 1 Correlation Space, 2001, ApJ, 558, pp. 553560

Marziani, P., Sulentic, J. W., Zamanov, R., et al., An Optical Spectroscopic Atlas of Low-Redshift Active Galactic Nuclei, 2003a, ApJS, 145, pp. 199211 
Marziani P., Zamanov R. K., Sulentic J. W., et al., Searching for the physical drivers of eigenvector 1: influence of black hole mass and Eddington ratio, 2003b, MNRAS, 345, pp. 1133-1144

Marziani, P., Sulentic, J. W., Stirpe, et al., VLT/ISAAC spectra of the H $\beta$ region in intermediate-redshift quasars. III. $\mathrm{H} \beta$ broad-line ile analysis and inferences about BLR structure, 2009, A\&A, 495, pp. 83-112

Matsuoka, Y., Oyabu, S., Tsuzuki, Y., et al., Observations of O I and Ca II Emission Lines in Quasars: Implications for the Site of Fe II Line Emission, 2007, ApJ, 663, pp. 781-798

Matsuoka, Y., Peterson, B. A., Oyabu, S., et al., Optical to Near-Infrared Spectrum of a Massive Evolved Galaxy at $\mathrm{z}=1.26$, 2008, ApJ, 685, pp. $767-772$

Moorwood, A., Cuby, J. G., Biereichel, P., et al., ISAAC sees first light at the VLT, 1998, The Messenger, 94, pp. 7-9

Osterbrock, Donald E. \& Ferland, Gary F., 2005, Astrophysics Of Gaseous Nebulae And Active Galactic Nuclei 2nd edition, University Science Books, Sausalito California.

Persson S. E., Calcium infrared triplet emission in active galactic nuclei, 1988, ApJ, 330, pp. 751-765

Rayner, J. T., Cushing, M. C., Vacca, W. D., The Infrared Telescope Facility (IRTF) Spectral Library: Cool Stars, 2009, ApJS, 185, pp. 289-432

Sánchez-Blázquez, P., Gorgas, J., Cardiel, N., et al., Stellar populations of early-type galaxies in different environments. I. Line-strength indices. Relations of line-strengths with $\sigma, 2006$, A\&A, 457, pp. 787-808

Sánchez-Blázquez, P., Gorgas, J., Cardiel, N., et al., Stellar populations of early-type galaxies in different environments. II. Ages and metallicities, 2006, A\&A, 457, pp. 809-821

Sánchez-Blázquez, P., Gorgas, J., Cardiel, N., Stellar populations of earlytype galaxies in different environments. III. Line-strength gradients, 2006, A\&A, 457, pp. 823-839 
Schlegel, D., Finkbeiner D. \& Davis M., Maps of Dust Infrared Emission for Use in Estimation of Reddening and Cosmic Microwave Background Radiation Foregrounds, 1998, ApJ, 500, pp. 525-553

Sulentic, J. W., Marziani, P. \& Dultzin-Hacyan, D., Phenomenology of Broad Emission Lines in Active Galactic Nuclei, 2000, ARA\&A, 38, pp. 521-571

Sulentic J. W., Zwitter T., Marziani P., et al., Eigenvector 1: An Optimal Correlation Space for Active Galactic Nuclei, 2000, ApJ, 536, pp. 5-9

Sulentic, J. W., Marziani, P., Zamanov, R., et al., Average Quasar Spectra in the Context of Eigenvector 1, 2002, ApJ, 566, pp. 71-75

Sulentic, J. W., Stirpe, G. M., Marziani, P.,et al., VLT/ISAAC spectra of the $\mathrm{H} \beta$ region in intermediate redshift quasars, 2004, A\&A, 423, pp. 121-132

Sulentic J. W., Bachev R., Marziani P., et al., C IV $\lambda 1549$ as an Eigenvector 1 Parameter for Active Galactic Nuclei, 2007, ApJ, 666, pp. 757-777 

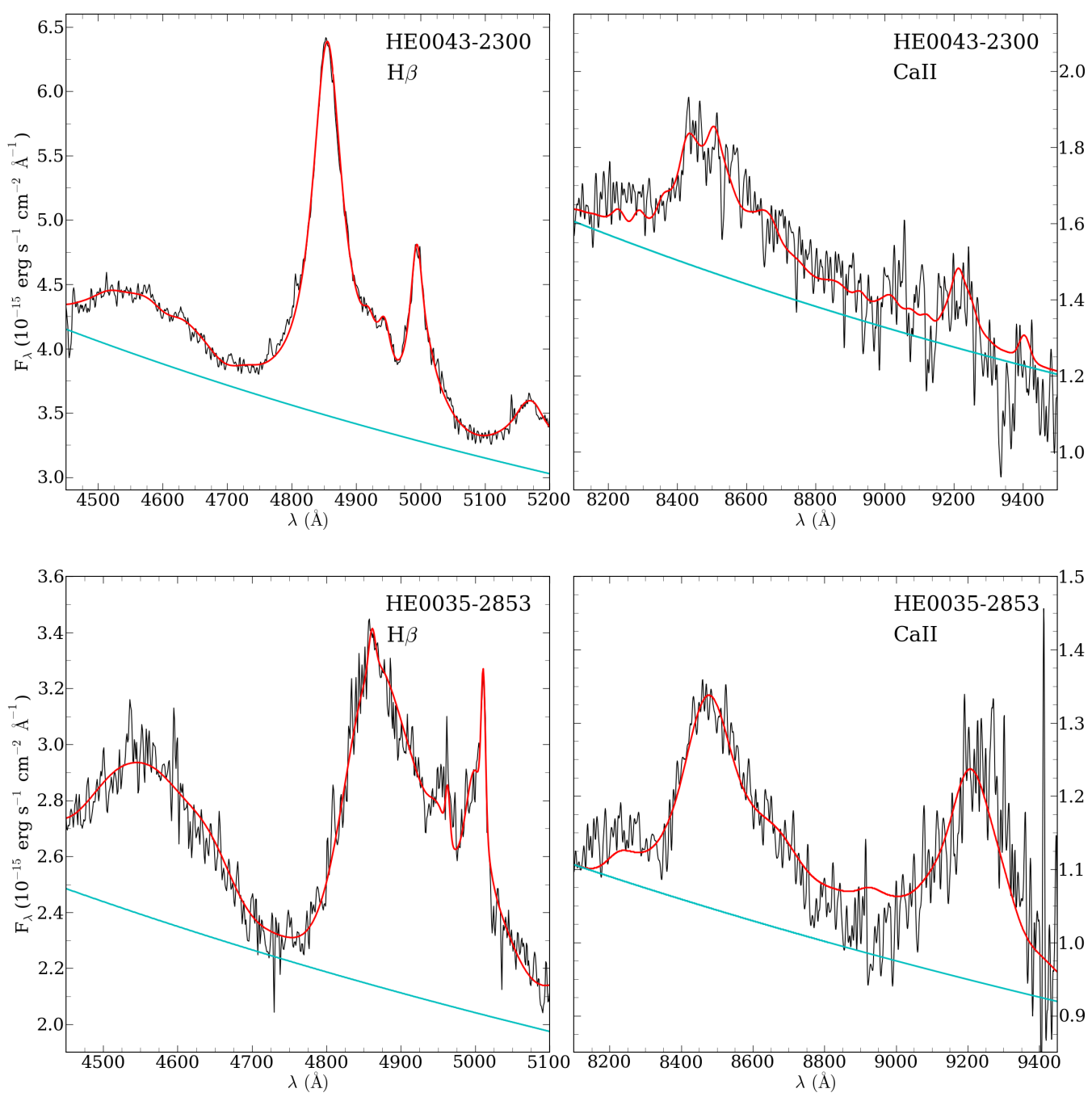

Figure 1: Example of intermediate-redshift quasars. The top panel shows a source representative of Pop. A, the bottom one of Pop. B. Abscissae are rest-frame wavelength in $\AA$, ordinates are specific flux in units of $10^{-15} \mathrm{erg} \mathrm{s}^{-1} \mathrm{~cm}^{-2} \mathrm{~A}^{-1}$. The left panels show $\mathrm{H} \beta$ spectral region before continuum subtraction. Right panels show CaII spectral region. In both panels the best fit is marked by the red color line and the continuum level is marked with the cyan line. 

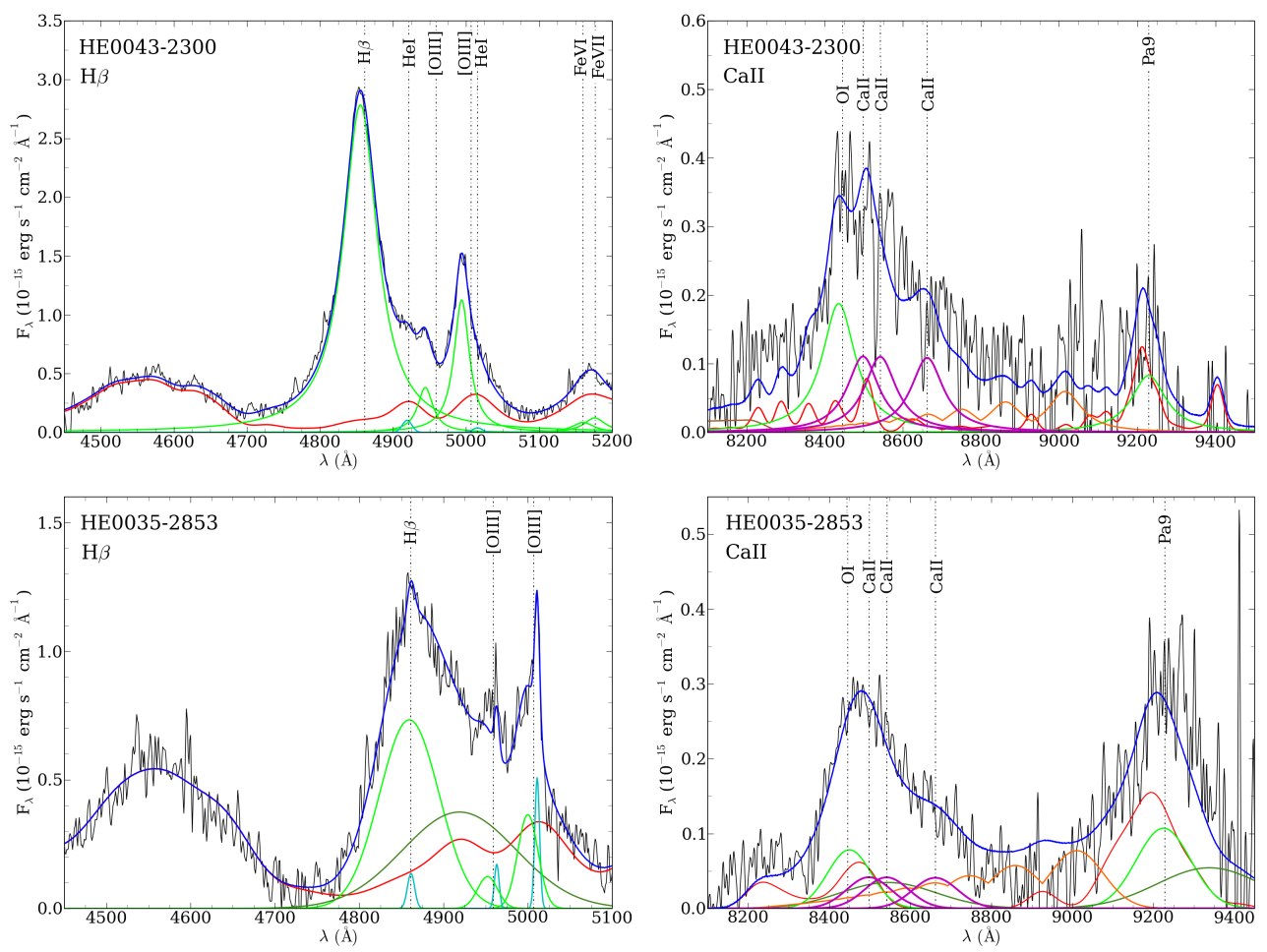

Figure 2: Continuum-subtracted spectra for $\mathrm{H} \beta$ and CaII spectral regions (left and right panels). Blue lines show the best fit. BC is marked by the fluorescent green, VBC is marked by the green line, $\mathrm{NC}$ is marked by the cyan line and the magenta line marks the CaII triplet. Red line traces FeII emission and orange line traces the pseudo-continuum of High Order Paschen Lines. The vertical dash-point lines mark the rest-frame wavelenght of the lines fitted. Abscissae are rest-frame wavelength in $\AA$, ordinate are specific flux in units of $10^{-15} \mathrm{erg} \mathrm{s}^{-1} \mathrm{~cm}^{-2} \AA^{-1}$. In $\mathrm{H} \beta$ spectra can be observed [OIII] $\lambda \lambda 5007,4959$, both spectra present blueshifted broad component commonly asociated to a wind. Only HE0035-2853 present a narrow component the rest-frame for $\mathrm{H} \beta$ and [OIII]. In HE00432300 could be observed a weak emission of HeI $\lambda 4921$, HeI $\lambda 5015$, FeVI $\lambda 5160$ and FeVII $\lambda 5177$. In the infrared spectra for both cases, OI and CaII triplet are completely blended, with OI most intense than CaII. In HE0035-2853 high order Paschen lines contribute largerly to the total spectrum. 


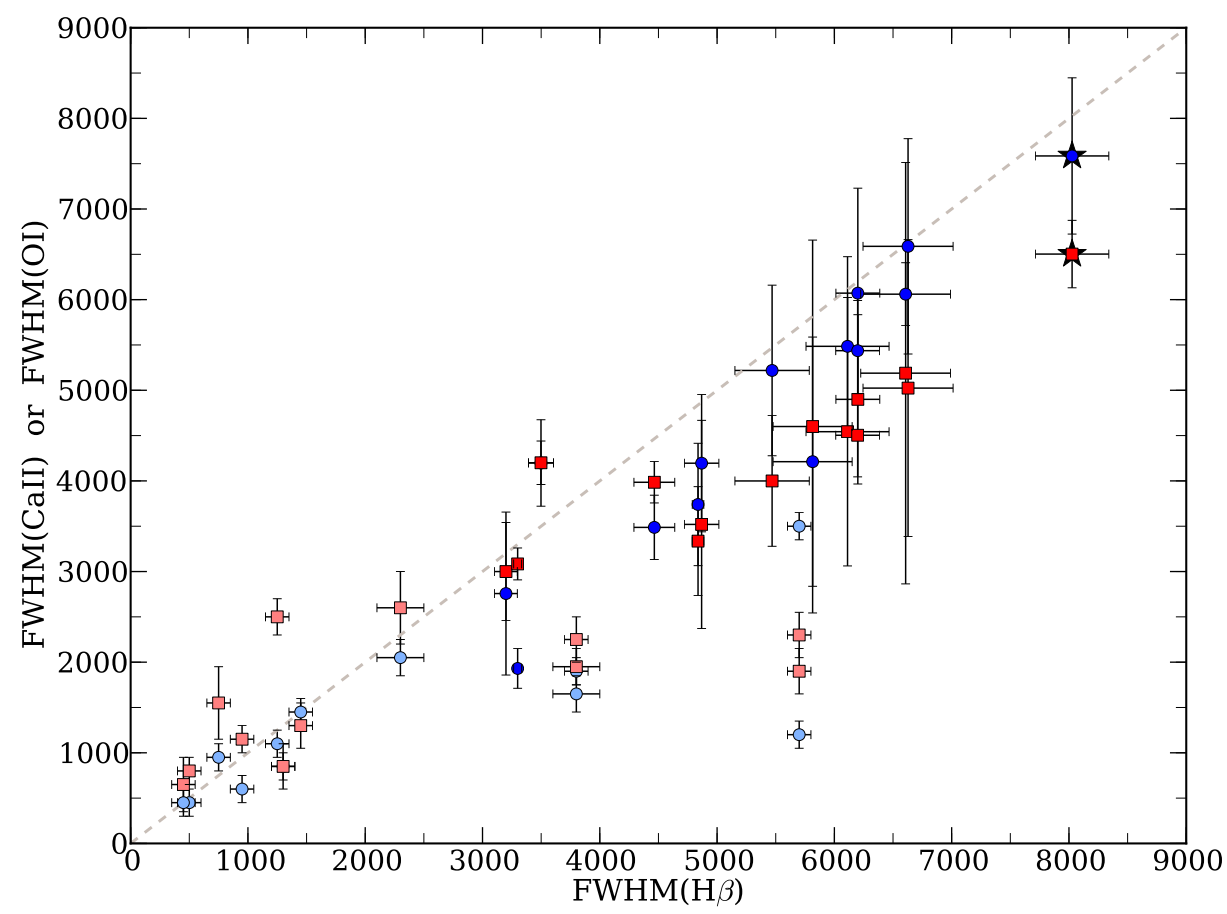

Figure 3: Comparison between CaII (BC), OI (BC+VBC) and $\mathrm{H} \beta(\mathrm{BC}+\mathrm{VBC}) \mathrm{FWHMs}$ in $\mathrm{km} \mathrm{s}^{-1}$. Abscissa is the $\mathrm{H} \beta \mathrm{FWHM}$, ordinate is the CaII Triplet and OI $\lambda 8446 \mathrm{FWHM}$. Blue circles: OI data. Red squares: CaII data; pale color data points belong to the Persson's sample (Persson, 1988). Starred point is HE2202-2557, the quasar with significant stellar absorption. The dashed line has a slope of unity. 
Table 1: Basic properties of sources

\begin{tabular}{cccccc}
\hline Target name & $\mathrm{z}^{1}$ & $\mathrm{M}_{B}{ }^{2}$ & $\log \left(\mathrm{R}_{K}\right)^{3}$ & Sp. T. $^{4}$ & Standard Star $^{5}$ \\
\hline \hline HE0005-2355 & 1.412 & -27.6 & 2.56 & $\mathrm{~B} 1$ & Hip000183 \\
HE0035-2853 & 1.638 & -28.1 & $<0.21$ & $\mathrm{~B}$ & Hip005988 \\
HE0043-2300 & 1.540 & -27.9 & 2.03 & $\mathrm{~A} 1$ & Hip005988 \\
HE0048-2804 & 0.847 & -26.0 & $\ldots$ & $\mathrm{B} 1$ & Hip005988 \\
HE0058-3231 & 1.582 & -27.9 & $<0.24$ & $\mathrm{~B} 1$ & Hip005988 \\
HE0203-4627 & 1.438 & -27.5 & 2.07 & $\mathrm{~B} 2$ & Hip012248 \\
HE0248-3628 & 1.536 & -28.2 & 0.55 & $\mathrm{~A} 1$ & Hip108612 \\
HE1349+0007 & 1.444 & -28.0 & -0.18 & $\mathrm{~B}$ & Hip082037 \\
HE1409+0101 & 1.650 & -28.3 & 0.40 & $\mathrm{~B}$ & Hip088609 \\
HE2147-3212 & 1.543 & -28.2 & $<0.14$ & $\mathrm{~B}$ & Hip099286 \\
HE2202-2557 & 1.535 & -28.1 & 1.80 & $\mathrm{~B} 1$ & Hip111563 \\
HE2340-4443 & 0.922 & -26.3 & $\ldots$ & A1 & Hip111563 \\
HE2349-3800 & 1.604 & -27.4 & 1.93 & B2 & Hip104374 \\
HE2352-4010 & 1.580 & -28.8 & $\ldots$ & A1 & Hip001904 \\
\hline
\end{tabular}

${ }^{1}$ Redshift, uncertain and reference in Sulentic et al. (2007) \& Marziani et al. (2009)

${ }^{2}$ Absolute magnitude, reference in Sulentic et al. (2007) \& Marziani et al. (2009)

${ }^{3}$ Decimal logarithm of the specific flux at $6 \mathrm{~cm}$ and $4440 \AA$

${ }^{4}$ Spectral type in the Eigenvector 1 sequence: Population A or B.

${ }^{5}$ Standard star name used for sensitivity function calibration. 